\title{
МУЛЬТИДИСЦИПЛИНАРНЫЙ ПОДХОД К ЛЕЧЕНИЮ НЕОРГАННЫХ ЗАБРЮШИННЫХ ОПУХОЛЕЙ
}

\author{
А. В. Скумс, В. А. Кондратюк, С. В. Сухачев, О. Н. Егорова, О. М. Симонов, А. А. Скумс \\ Национальный институт хирургии и трансплантологии имени А. А. Шалимова НАМН Украины, г. Киев

\section{MULTIDISCIPLINARY APPROACH TO TREATMENT OF EXTRAORGAN RETROPERITONEAL TUMOURS}

\author{
A. V. Skums, V. A. Kondratyuk, S. V. Sukhachev, O. N. Egorova, O. M. Simonov, A. A. Skums \\ Shalimov National Institute of Surgery and Transplantology, Kiev
}

\begin{abstract}
Реферат
Проанализированы результаты хирургического лечения 11 пациентов по поводу неорганных забрюшинных опухолей (НЗО) с использованием мультидисциплинарного подхода. Тактика лечения предусматривала последовательные этапы: мультиспиральную компьютерную томографиюю (МСКТ) с трехмерной реконструкцией сосудов, одной из целей которой является определение источника кровоснабжения опухоли; рентгеноэндоваскулярную селективную эмболизацию сосудов, питающих опухоль; хирургическое удаление опухоли. Предложенная тактика хирургического лечения НОЗ соответствует онкологическим принципам и позволяет минимизировать интраоперационную кровопотерю.

Ключевые слова: неорганная забрюшинная опухоль; мультивисцеральная резекция; мультидисциплинарный подход.

Abstract

Results of surgical treatment of 11 patients, suffering extraorgan retroperitoneal tumors, using multidisciplinary approach, were analyzed. Tactics of treatment have included subsequent stages: multispiral computeric tomography (MSCT) with three-dimensional reconstruction of the vessels, aiming, in particular, to determine the tumor blood supply origin; roentgenoendovascular selective embolization of vessels, which nourish the tumor; surgical resection of the tumor. The tactics proposed for surgical treatment of extraorgan retroperitoneal tumors answers oncological principles and permits to make intraoperative blood loss minimal.

Keywords: extraorgan retroperitoneal tumour; multivisceral resection; multidisciplinary approach.
\end{abstract}

Неорганные забрюшинные опухоли (НЗО) - достаточно редкое заболевание, составляют 0,3 - $1 \%$ в структуре всех новообразований [1 $-3]$.

В 20\% наблюдений НЗО являются доброкачественными, в 80\% - злокачественными [4]. Единственный метод радикального лечения НЗО - хирургический, лучевая терапия и химиотерапия недостаточно эффективны [5]. Радикальное удаление новообразований забрюшинного пространства сопряжено с угрозой возникновения массивного и сверхмассивного (до 70\% объема циркулирующей крови) кровотечения, повреждения жизненно важных органов; оно относится к числу высоко травматичных вмешательств с высокой степенью операционного риска $[6,7]$.

Целью исследования был поиск возможностей уменьшения риска возникновения интраоперационного кровотечения при удалении НЗО больших размеров.

\section{МАТЕРИАЛЫ И МЕТОДЫ ИССЛЕДОВАНИЯ}

За период 2015 - 2016 гг. по поводу Н3О с применением мультидисциплинарного подхода оперированы 11 больных, в том числе 8 женщин и 3 мужчин, в возрасте от 35 до 67 лет.

Тактика лечения включала последовательное выполнение следующих этапов.

1. МСКТ с трехмерной реконструкцией сосудов, одной из целей которой является определение источника кровоснабжения опухоли.

2. Рентгеноэндоваскулярная селективная эмболизация артерий, кровоснабжающих НЗО.

3. Оперативное вмешательство.

Обследование пациентов было комплексным, включало лабораторные и инструментальные методы (УЗИ, ЭГДФС, МСКТ, реносцинтиграфия, колоноскопия, ангиография).

По данным МСКТ с внутривенным контрастированием определя- ли не только размеры опухоли, ее расположение, взаимоотношение с соседними органами и структурами, но и источники кровоснабжения.

Следующим этапом было проведение ангиографии с применением доступа через бедренную или плечевую артерию и селективной рентгеноэндоваскулярной эмболизации артериальных сосудов, питающих опухоль.

На следующий день производили хирургическое удаление НЗО.

\section{РЕЗУЛЬТАТЫ}

При НЗО клинические симптомы неспецифические, нечетко выражены, опухоль чаще обнаруживали случайно. Больные жаловались на наличие ноющей боли в животе, общую слабость, иногда - субфебрильную температуру тела. Некоторые пациенты отмечали увеличение и асимметрию живота.

Основными задачами инструментального исследования были определение локализации и размеров 
опухоли, ее взаимоотношения с соседними органами и структурами, их возможной инвазии опухолью, степени васкуляризации, источников ее кровоснабжения. При обнаружении гиперваскуляризированной опухоли по данным МСКТ с использованием трехмерной реконструкции идентифицировали кровоснабжающие ее сосуды для целенаправленного выполнения ангиографии и эмболизации. При слабо и умеренно выраженной васкуляризации опухоли сосуды для эмболизации определяли с учетом данных МСКТ и ангиографии. При несомненных признаках инвазии опухолью соседних органов и необходимости их удаления или резекции устанавливали показания к проведению рентгеноэндоваскулярной окклюзии сосудов этих органов. На момент диагностики диаметр опухолей составлял $18-48$ см.

В целях деваскуляризации опухоли при предоперационной подготовке эмболизация внутренней подвздошной артерии и ее ветвей выполнена у 3 больных, надпочечниковых артерий - у 3, селезеночной артерии - у 3, нижней брыжеечной и крестцовой артерий - у 1, почечной артерии - у 1. У 3 больных выполнена эмболизация артерий (селезеночной - у 2, почечной - у 1) при доказанной инвазии опухолью поджелудочной железы или почки с учетом объема предстоящего вмешательства. Осложнений, связанных с процедурой, не было. Болевой синдром умеренной интенсивности, возникший у 2 больных, устранен путем введения нестероидных противовоспалительных препаратов.

На следующий день после рентгеноэндоваскулярного вмешательства осуществляли хирургическое удаление НЗО. Вариант операции с первоначальным выходом на питающие сосуды в связи с большими размерами опухоли был возможен только у одного больного, у остальных - вначале мобилизовали опухоль до достижения ее подвижности и определения взаимоотношений с окружающими органами и структурами. Этот этап операции выполняли с минимальной кровопотерей благодаря деартериализации опухо- ли. После тщательной ревизии принимали решение о необходимости выполнения и объеме мультивисцеральной резекции. Удаление забрюшинной опухоли осуществлено у 5 больных, в сочетании с нефрэктомией - у 2, дистальной резекцией поджелудочной железы и спленэктомией - у 2 , дистальной резекцией поджелудочной железы, спленэктомией, резекцией желудка, эпинефрэктомией - у 1, нефрэктомией, резекцией нижней полой вены и двенадцатиперстной кишки - у 1.

Объем кровопотери составлял от 100 до 200 мл, гемотрансфузия не потребовалась, все пациенты живы.

По данным гистологического исследования, липосаркома диагностирована у 5 пациентов, фибросаркома - у 3, лейомиосаркома, злокачественная гемангиоперицитома,

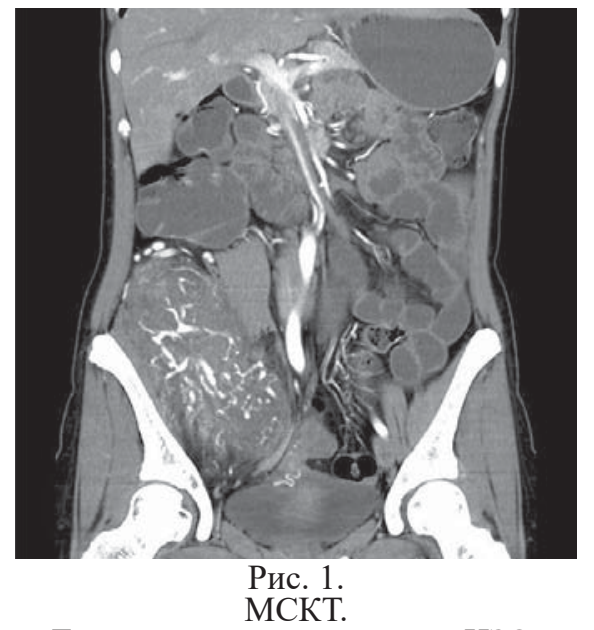

Гиперваскуляризированная НЗО.

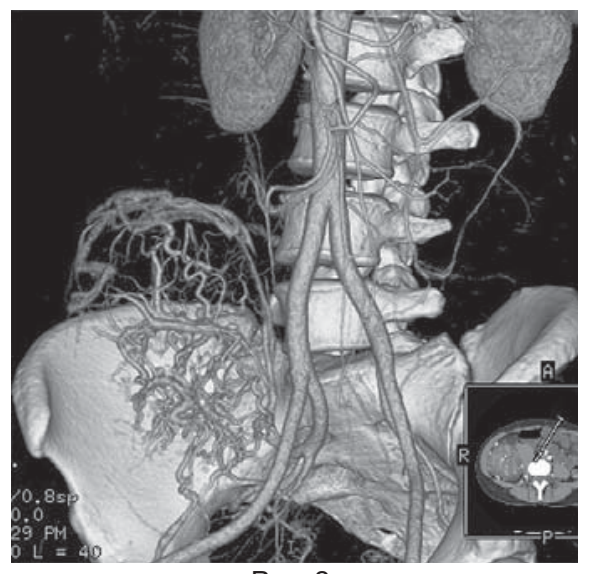

Рис. 2.

МСКТ с трехмерной реконструкцией сосудов.

Интенсивное кровоснабжение опухоли из правой внутренней подвздошной артерии. фиброма - по одному наблюдению. Приводим наблюдение.

Больная В., 35 лет, 18.01.2016 госпитализирована с жалобами на наличие большого, плотного, выпячивающегося через переднюю брюшную стенку опухолеподобного образования в правой паховой области. Считает себя больной с января 2007 г. Обследована в соответствии с алгоритмом.

По данным МСКТ, в правой паховой области определяется образование плотной консистенции, размерами $18 \times 12$ см, расположенное на подвздошной кости и крестце справа, распространяется вниз до бедренного канала, оттесняет кпереди поясничные мышцы и подвздошные сосуды. Опухоль имеет обширную сеть патологически-измененных сосудов. Заключение: НЗО (рис. 1).

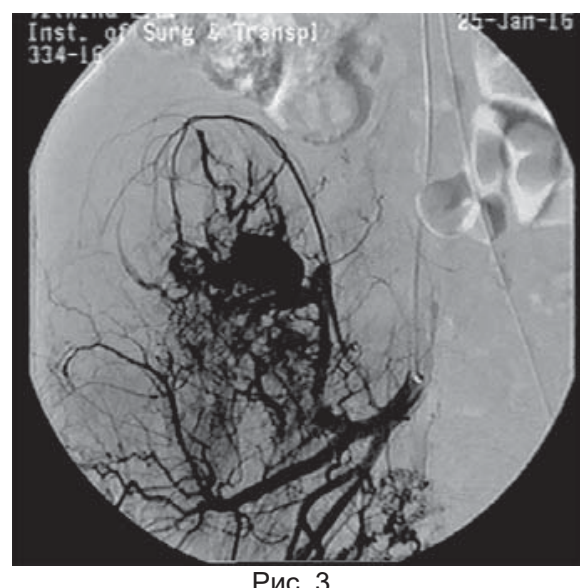

Ангиографрия внутренней подвздошной артерии.

Гиперваскуляризированная НЗО.

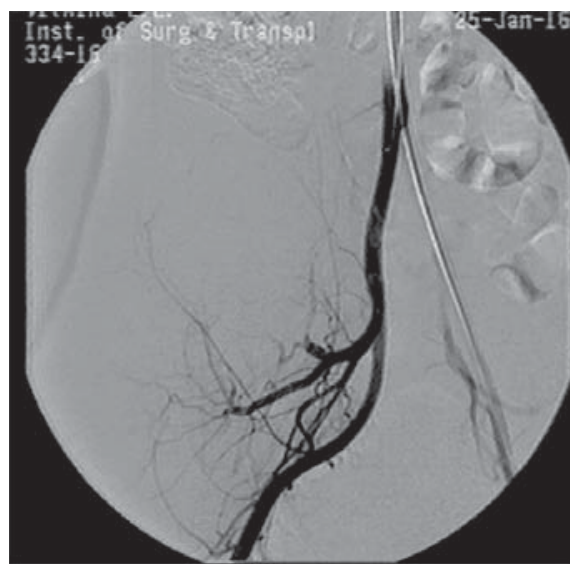

Рис. 4.

Ангиография после эмболизации ветвей внутренней подвздошной артерии. Редукция артериального кровотока в опухоли. 
По результатам МСКТ с трехмерной реконструкцией сосудов обнаружено интенсивное кровоснабжение опухоли, установлен его источник - ветви правой внутренней подвздошной артерии (рис. 2).

Во время предоперационной подготовки решено осуществить эндоваскулярную эмболизацию питающих сосудов. Доступом через левую бедренную артерию произведена катетеризация правой общей подвздошной артерии. По данным ангиографии подтверждено интенсивное кровоснабжение опухоли из ветвей правой внутренней подвздошной артерии (рис. 3). Поочередно произведены селективная катетеризация и эмболизация артериальных ветвей, кровоснабжающих опухоль, металлическими спиралями и поролоновыми эмболами. При контрольной ангиографии уже к моменту окончания процедуры отмечено значительное обеднение сосудистого рисунка опухоли (рис. 4).

На следующий день выполнена операция - удаление НЗО.

При ревизии забрюшинного пространства справа обнаружена неподвижная опухоль размерами $18 \times$ 12 см, спаянная с надкостницей подвздошной кости, распространяющаяся вниз до бедренного канала. Поясничные мышцы, наружные подвздошные сосуды, мочеточник и седалищный нерв смещены кпереди и влево.

Опухоль поэтапно выделена из окружающих тканей, окклюзированные артериальные ветви легко обнаружены и пересечены. Объем кровопотери составил 160 мл. Удаленный макропрепарат - десмоидная фиброма.

Течение послеоперационного периода без осложнений. Пациентка выписана в удовлетворительном состоянии на 8-е сутки после операции.

\section{ОБСУЖДЕНИЕ}

Следует подчеркнуть, что по поводу НЗО не существует стандартных оперативных вмешательств, однако возможны два варианта мобилизации опухоли [8]. Первый вариант более предпочтительный - выход на питающие опухоль сосуды, их перевязка, пересечение и затем почти бескровное удаление опухоли. Такой подход более оправдан с онкологической точки зрения для предупреждения интраоперационной диссеминации клеток опухоли. Как правило, это выполнимо при опухолях диаметром не более 10 см, с определенной степенью подвижности, при условии четкого представления (на основании дооперационных данных и результатов ревизии) о наличии «питающего» сосуда и взаимоотношениях с предлежащими структурами. Второй вариант мобилизации предполагает последовательное выделение наиболее свободных от взаимосвязи с соседними анатомическими структурами участков опухоли. После того, как опухоль становится подвижной, возможно определить ее взаимоотношение с соседними органами и источник кровоснабжения. Таким образом, сосуды лигируют и пересекают в последнюю очередь. Заднюю поверхность опухоли, как правило, выделяют без адекватного визуального контроля, поэтому остановка возникшего кровотечения в условиях ограниченного операционного поля представляет серьезную проблему.

Для НЗО характерны медленный рост и отсутствие специфических симптомов. Это, а также анатомические особенности забрюшинного пространства, обусловливают выявление опухолей уже значительных размеров (у 80\% больных диаметром более 10 см) с вовлечением смежных органов [9]. По данным литературы, диаметр выявленных опухолей составил от 4 до 41 см, в среднем 17,5 см, только у 2 больных он не превышал 5 см [10]. Другие авторы обнаруживали опухоли диаметром от 14 до 43 см, в среднем (21,3 $\pm 4,4)$ см [6]. Некоторые авторы выявляли опухоли диаметром от 10 до 60 см, небольших опухолей (диаметром менее 10 см) не наблюдали, от 10 до 20 см - у 15,8\% больных. У 16 (84,2\%) больных диаметр новообразования превышали 20 см, в среднем составил $(34,16 \pm 13,3)$ см [11]. Таким образом, в большинстве наблюдений НЗО диагностировали при их диаметре более 10 см.
Важным фактором, определяющим возможность возникновения тяжелого кровотечения, считают степень васкуляризации опухоли. Авторы напрямую связывают объем кровопотери со степенью васкуляризации опухоли [11]. Наименьший (1567 мл) объем кровопотери отмечали при аваскулярном типе опухоли; при слабо и умеренно выраженной васкуляризации опухоли он составил соответственно 1732 и 1927 мл; при выраженном кровоснабжении опухоли кровопотеря была почти в 2,5 раза больше (3234 мл). К гиперваскуляризированным опухолям относят гемангиоперицитому и параганглиому, к умеренно васкуляризированным - злокачественную фиброзную гистиоцитому, лейомиосаркому и другие виды саркомы; липосаркома, лимфома и многие доброкачественные опухоли характеризуются слабой васкуляризацией [12].

Очевидно, при выполнении мультивисцеральной резекции риск кровопотери также увеличивается. По данным литературы, ее выполняют у 34 - 93\% больных $[5,13,14]$. Так, мультивисцеральная резекция потребовалась у 50\% больных (нефрэктомия - у 35\%, резекция толстой кишки - у $21 \%$, спленэктомия - у $10 \%$, резекция поджелудочной железы - у 9\%, магистральных сосудов и др.) [10]. Комбинированные хирургические вмешательства авторы выполняли с резекцией одного органа у 46,4\% больных, двух органов - у 26,6\%, трех органов - у 15,1\%, четырех и более органов - у 12\% [11]. Максимальное число резецированных органов - 5 . Наиболее часто выполняли нефрэктомию (у 47\% больных), спленэктомию (у 19\%), резекцию тонкой и ободочной кишки (у 18 и 24\%), тела и хвоста поджелудочной железы (у 18\%); реже - резекцию диафрагмы (у 12\%), печени (у 10\%), желудка (у 5\%). Объем кровопотери при комбинированном удалении НЗО составил в среднем 2347 мл, при операциях без резекции смежных органов 978 мл. В некоторых ситуациях, при больших размерах опухоли с вовлечением прилежащих органов и сосудистых структур, объем кровопотери достигал 8500 мл. 
Таким образом, тактика хирургического лечения НЗО с предварительной эмболизацией сосудов имеет теоретическое и практическое обоснование. С позиций современной онкологии, хирургическое лечение НЗО должно быть мультидисциплинарным. Первоначальное прекращение артериального кровотока в опухоли до ее мобилизации соответствует онкологическим принципам выполнения операций.

\section{ЛITEPATYPA/REFERENCES}

1. Boyko VV, Dalavurak VP, Savvy SA, ta in. Neorgannye opukholy zabriushynnogo prostranstva: dyagnostyka i khirurhycheskoe lechenie. Kharkivska khirurhichna shkola. 2011;4(49):26-31. [In Russian].

2. Brennan C, Kajal D, Khalili K, Ghai S. Solid malignant retroperitonea masses-a pictorial review. Insights Imaging. 2014;(5):53-65.

3. Vickie Y, Christopher D. WHO classification of soft tissue tumours: an update based on the 2013 (4th) edition. Pathology. 2014;46(2):95104.

4. Lazar A, Brãtucu E, Straja N, et al. Primitive retroperitoneal tumors. Vascular involvement a major prognostic factor. Chirurgia. 2012;107(2):186-94.

5. Strauss D, Hayes A, Thomas J. Retroperitoneal tumours: review of management. Ann R Coll Surg Engl. 2011;(93):275-80.

6. Qin $Y$, Yang $Y$, Yang $H$, Li J. Curved incision under the costal margin in retroperitoneal tumor surgery. Molecular and Clinical Oncology. 2015;(3):789-92.

7. Ikeguchi M, Urushibara S, Shimoda R. Surgical treatment of retroperitoneal liposarcoma. Yonago Acta Medica. 2014;57(4):129-32.

8. Klimenkov A, Gubina G. Neorgannye zabryushinnye opukholi: osnovnye printsipy diagnostiki i khirurgicheskol taktiki. Prakticheskaya onkologiya. 2004;5(4):285-90. [In Russian].
9. Mullinax J, Zagr J, Gonzalez R. Current diagnosis and management of retroperitoneal sarcoma. Cancer Control. 2011;18(3):177-87.

10. Gholami S, Jacobs C, Kapp D, et al. The value of surgery for retroperitoneal sarcoma [Internet]. Hindawi Publishing Corporation Sarcoma Volume. 2009. Режим доступу до ресурсу: doi:10.1155/2009/605840.

11. Davydov M. Strategiya khirurgii vneorgannykh sarkom torakoabdominal'no lokalizatsii [dissertatsiya]. Moskva, 2015. 303 c. [In Russian].

12. Nishino M, Hayakawa K, Minami M, et al. Primary retroperitoneal neoplasms: CT and MR imaging findings with anatomic and pathologic diagnostic clues. Radio Graphics. 2003;(23):45-57.

13. Gronchi A, Lo Vullo S, Fiore M. Aggressive surgical policies in a retrospectively reviewed single-institution case series of retroperitoneal soft tissue sarcoma patients. J Clin Oncol. 2009;27:24-30.

14. Lehnert T, Cardona S, Hinz U. Primary and locally recurrent retroperitoneal soft-tissue sarcoma: local control and survival. Eur J Surg Oncol. 2009;(35):986-93. 